Chapman University

Chapman University Digital Commons

Pharmacy Faculty Articles and Research

School of Pharmacy

4-28-2013

\title{
Liposomal Fasudil, a Rho-Kinase Inhibitor, for Prolonged Pulmonary Preferential Vasodilation in Pulmonary Arterial Hypertension
}

Vivek Gupta

Keck Graduate Institute, vivek_gupta@kgi.edu

Nilesh Gupta

Texas Tech University Health Sciences Center

Imam H. Shaik

Texas Tech University Health Sciences Center

Reza Mehvar

Chapman University, mehvar@chapman.edu

Ivan F. McMurty

University of South Alabama

See next page for additional authors

Follow this and additional works at: http://digitalcommons.chapman.edu/pharmacy_articles

Part of the Cardiovascular Diseases Commons, Pharmaceutics and Drug Design Commons, and the Pharmacology Commons

\section{Recommended Citation}

Gupta, Vivek, Nilesh Gupta, Imam H. Shaik, Reza Mehvar, Ivan F. McMurtry, Masahiko Oka, Eva Nozik-Grayck, Masanobu Komatsu, and Fakhrul Ahsan. "Liposomal fasudil, a rho-kinase inhibitor, for prolonged pulmonary preferential vasodilation in pulmonary arterial hypertension." Journal of Controlled Release 167, no. 2 (2013): 189-199.

DOI:10.1016/j.jconrel.2013.01.011

This Article is brought to you for free and open access by the School of Pharmacy at Chapman University Digital Commons. It has been accepted for inclusion in Pharmacy Faculty Articles and Research by an authorized administrator of Chapman University Digital Commons. For more information, please contact laughtin@chapman.edu. 


\section{Liposomal Fasudil, a Rho-Kinase Inhibitor, for Prolonged Pulmonary Preferential Vasodilation in Pulmonary Arterial Hypertension}

\section{Comments}

NOTICE: this is the author's version of a work that was accepted for publication in Journal of Controlled Release. Changes resulting from the publishing process, such as peer review, editing, corrections, structural formatting, and other quality control mechanisms may not be reflected in this document. Changes may have been made to this work since it was submitted for publication. A definitive version was subsequently published in Journal of Controlled Release, volume 167, issue 2, in 2013. DOI: 10.1016/j.jconrel.2013.01.011

The Creative Commons license below applies only to this version of the article.

\section{Creative Commons License}

\section{(c) (1) (8)}

This work is licensed under a Creative Commons Attribution-Noncommercial-No Derivative Works 4.0 License.

\section{Copyright}

Elsevier

\section{Authors}

Vivek Gupta, Nilesh Gupta, Imam H. Shaik, Reza Mehvar, Ivan F. McMurty, Masahiko Osa, Eva NozikGrayck, and Fakhrul Ahsan 


\title{
Liposomal Fasudil, a Rho-Kinase Inhibitor, for Prolonged Pulmonary Preferential Vasodilation in Pulmonary Arterial Hypertension
}

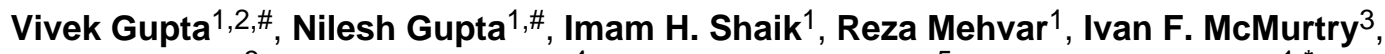 \\ Masahiko Oka ${ }^{3}$, Eva Nozik-Grayck ${ }^{4}$, Masanobu Komatsu ${ }^{5}$, and Fakhrul Ahsan ${ }^{1, *}$ \\ ${ }^{1}$ Department of Pharmaceutical Sciences, School of Pharmacy, Texas Tech University Health \\ Sciences Center, 1300 S Coulter, Amarillo, TX - 79106, USA \\ ${ }^{3}$ Departments of Pharmacology and Medicine, The Center for Lung Biology, University of South \\ Alabama, Mobile, AL - 36688, USA \\ ${ }^{4}$ Department of Pediatrics, University of Colorado Denver, Anschutz Medical Campus, Aurora, \\ CO - 80045, USA \\ ${ }^{5}$ Sanford-Burnham Medical Research Institute at Lake Nona, Orlando, Florida - 32827, USA
}

\section{Abstract}

Current pharmacological interventions for pulmonary arterial hypertension (PAH) require continuous infusions, multiple inhalations, or oral administration of drugs that act on various pathways involved in the pathogenesis of PAH. However, invasive methods of administration, short duration of action, and lack of pulmonary selectivity result in noncompliance and poor patient outcomes. In this study, we tested the hypothesis that encapsulation of an investigational anti-PAH molecule fasudil (HA-1077), a Rho-kinase inhibitor, into liposomal vesicles results in prolonged vasodilation in distal pulmonary arterioles. Liposomes were prepared by hydration and extrusion method and fasudil was loaded by ammonium sulfate-induced transmembrane electrochemical gradient. Liposomes were then characterized for various physicochemical properties. Optimized formulations were tested for pulmonary absorption and their pharmacological efficacy in a monocrotaline (MCT) induced rat model of PAH. The entrapment efficiency of optimized liposomal fasudil formulations was between $68.1 \pm 0.8 \%$ and $73.6 \pm 2.3 \%$, and the cumulative release at $37^{\circ} \mathrm{C}$ was $98-99 \%$ over a period of 5 days. Compared to intravenous (IV) fasudil, a $~ 10$ fold increase in the terminal plasma half-life was observed when liposomal fasudil was administered as aerosols. The $\mathrm{t}_{1 / 2}$ of IV fasudil was $0.39 \pm 0.12 \mathrm{~h}$. and when given as liposomes via pulmonary route, the $\mathrm{t}_{1 / 2}$ extended to $4.71 \pm 0.72 \mathrm{~h}$. One $\mathrm{h}$ after intratracheal instillation of liposomal fasudil, mean pulmonary arterial pressure (MPAP) was reduced by $37.6 \pm 5.7 \%$ and continued to decrease for about $3 \mathrm{~h}$, suggesting that liposomal formulations produced pulmonary preferential vasodilation in MCT induced PAH rats. Overall, this study established the proof-of-principle that aerosolized liposomal fasudil is a feasible option for a noninvasive, controlled release and pulmonary preferential treatment of PAH.

(C) 2012 Elsevier B.V. All rights reserved.

"To whom correspondence should be addressed. Phone: 806-356-4015 (Ext. 335), Fax: 806-356-4034, fakhul.ahsan@ttuhsc.edu. \#Equal Contributions

${ }^{2}$ Current Address: Department of Chemical Engineering, University of California, Santa Barbara, CA - 93106, USA

Publisher's Disclaimer: This is a PDF file of an unedited manuscript that has been accepted for publication. As a service to our customers we are providing this early version of the manuscript. The manuscript will undergo copyediting, typesetting, and review of the resulting proof before it is published in its final citable form. Please note that during the production process errors may be discovered which could affect the content, and all legal disclaimers that apply to the journal pertain. 


\section{Keywords}

Fasudil; liposomes; ammonium sulfate; pulmonary delivery; pulmonary arterial pressure

\section{INTRODUCTION}

Current therapeutic intervention for pulmonary arterial hypertension (PAH), a debilitating disorder of the pulmonary circulation, involves use of various categories of drugs that include prostacyclin analogues, endothelin receptor antagonists (ERAs), nitric oxide (NO) and phosphodiesterase-5 (PDE-5) inhibitors [1]. Unfortunately, none of these medications is optimal with regard to ease of administration, pulmonary selectivity, drug stability, safety, and efficacy. Systemic exposure produces many off-target effects, including peripheral vasodilation and reduced cardiac function. Consequently, existing anti-PAH therapy fails to prevent progression of the disease, and patient morbidity and mortality remain unacceptably high [2]. In fact, an inhalable formulation that can deliver drugs directly to the lungs at a reduced dosing frequency would address many limitations associated with current drug delivery approach for the treatment of PAH. Indeed, based on this assumption, we and others have attempted to use polymeric nano- or microparticles, liposomes and nanomicelles for inhalational therapy of PAH [3-5]. Further, aerosolized PEG-PLGA nanoparticles containing nuclear factor kappa-B (NF- $\kappa \mathrm{B}$ ) oligodeoxynucleotides (ODNs) reduced pulmonary arterial remodeling [4], and nanoparticles of pitavastatin exhibited vasculoprotective effects [3] in MCT induced PAH rats.

Similar to polymeric particles, liposomes have also been studied for their potential in inhalation therapy $[6,7]$. As inhalational carriers, liposomes are well tolerated by the lungs because lipids used to prepare liposomes dissolves readily in the respiratory fluid and serve as pulmonary surfactants [8]. Further, liposomes are stable lipidic carriers because particles in liposomal system are kinetically entrapped rather than thermodynamically equilibrated [9]. For many years, liposomes have been used for the delivery of antimicrobial agents to the lungs [10] and various peptides and small molecular weight drugs to produce systemic effects [11]. Liposomes have also been used for inhalational delivery of anti-PAH drugs such as iloprost [12] and vasoactive peptides [13].

Recently, Rho-kinase inhibitors have emerged as a new class of drugs with encouraging potential for the treatment of PAH. Fasudil, a Rho-kinase inhibitor, has been found to produce pulmonary vasodilation in both animal model and human PAH [14]. This drug alleviates the symptoms of PAH by eliciting pulmonary vasodilation and down-regulating the expression of growth factors, cell proliferation markers, and matrix proteins, and upregulating the expression of apoptotic markers. However, similar to prostacyclins, intravenous fasudil is not selective for the pulmonary circulation and has a half-life of $\sim 45$ minutes [15]. Although inhalation of this new anti-PAH therapeutic agent has shown potential for pulmonary selective vasodilation, there is no report regarding development of a long-lasting inhalational formulation for fasudil. Currently, it is not known whether fasudil encapsulated in liposomes will be effective in the treatment of PAH. In this study, we sought to test the hypothesis that fasudil encapsulated in aerosolized liposomes is a viable and efficacious approach for producing prolonged pulmonary selective vasodilation in PAH. In this regard, we have prepared fasudil liposomes, characterized their physical properties, investigated cellular uptake and tested their pharmacological efficacy in a rodent model of PAH. 


\section{MATERIALS AND METHODS}

\subsection{Materials}

Lipids, 1,2-dipalmitoyl-sn-glycero-3-phosphocholine (DPPC), and cholesterol from ovine wool (CHOL) were purchased from Avanti polar lipids, Inc. (Alabaster, Alabama, USA). Fasudil monohydrochloride and monocrotaline (MCT) were purchased from LC labs, Inc. (Woburn, MA, USA) and Sigma-Aldrich, Inc. (St. Louis, MO), respectively. SephadexG-25 PD-10 pre-packed column was purchased from GE Healthcare (Piscataway, New Jersey, USA). All other chemicals including chloroform, methanol, phosphate buffered saline (PBS 1X), ammonium sulfate, ammonium thiocyanate, ferric chloride, acetonitrile, dimethyl sulfoxide (DMSO), and perchloric acid were of analytical grade and obtained from various vendors in the United States. All chemicals were used without further purification.

\subsection{Preparation of liposomes}

Liposomes were prepared using DPPC:CHOL at a molar ratio of 7:3, and the total lipid concentration was $30 \mathrm{mM}$. Briefly, lipids were dissolved in a 4:1 mixture of chloroform and methanol and dried overnight at $40^{\circ} \mathrm{C}$ to form a thin film in a round bottom flask using a rotary evaporator (Buchi Rotor Evaporator R-114; BUCHI Labortechnik AG, Switzerland). The drug was incorporated either by passive or active loading. For passive loading, dried film was rehydrated with fasudil $(15 \mathrm{mg} / \mathrm{ml})$ in PBS and the rehydrated film was sonicated for $1 \mathrm{~h}$ at $25^{\circ} \mathrm{C}$ (Formulation F-1 in Table 1). Large multilamellar vesicles were extruded through LiposoFast ${ }^{\circledR}$ Extruder (Avestin, Inc., Canada) at $65^{\circ} \mathrm{C}$. Small unilamellar vesicles (SUVs) were collected following 21 cycles of extrusion from the opposite side of the extruder. Unencapsulated drug was removed by passing drug loaded liposomes through Sephadex-G-25 PD-10 column equilibrated with PBS (1×, $\mathrm{pH}$ 7.4). In case of active loading, dried lipid film was first rehydrated with $250 \mathrm{mM}$ ammonium sulfate $\left(\mathrm{NH}_{4}\right)_{2} \mathrm{SO}_{4}$ solution at varying $\mathrm{pH}(5.4,3.0,7.0$ and 8.0), and the resulting liposomes were extruded as described above and a transmembrane gradient was generated by exchanging external ammonium sulfate with ammonium ion free PBS solution using PD-10 column (Formulations F-2 to F-5). The drug was encapsulated by incubating it with ammonium sulfate entrapped liposomes for 30 minutes at $65^{\circ} \mathrm{C}$ and unentrapped drug was separated using the same column. Drug loaded liposomes were concentrated using Amicon Ultra centrifugal filter units (MWCO-3000, Millipore Inc., Billerica, Massachusetts, USA) by centrifugation for 45 min at 1500 rpm (Centrifuge 5702-R, Eppendorf AG, Hamburg, Germany). Liposomes thus prepared were stored at $4^{\circ} \mathrm{C}$ for future studies.

\subsection{Physicochemical characterization}

For determination of entrapment efficiency, liposomes (10 $\mathrm{\mu l})$ were lysed in methanol $(990 \mu \mathrm{l})$, sonicated, and centrifuged to separate the drug from the lipids. The amount of fasudil in supernatant was measured at $320 \mathrm{~nm}$ using a UV spectrophotometer (UV/Vis 918, GBC Scientific Equipment, Hampshire, Illinois, USA). The amount of lipid was determined from a standard curve prepared using phospholipid. The average vesicle size, polydispersity and zeta potential of the liposomes were determined using a Malvern Zetasizer (Malvern ${ }^{\circledR}$ Instruments Limited, Worcestershire, UK). The morphology of the liposomal formulations was studied by a Transmission Electron Microscope (TEM) (Hitachi H-7650, Hitachi High Technologies America, Inc., Pleasanton, CA). The in-vitro drug release studies were performed in dialysis cassettes (Slide-A-Lyzer, 3500 MWCO, 0.1-0.5 ml, ThermoScientific, Waltham, MA) as reported previously [16]. Briefly, the dialysis cassettes were first hydrated with PBS (pH 7.4) and $500 \mu$ of liposomes were loaded with a syringe. Plain fasudil was used as a control to evaluate whether dialysis cassettes themselves have any influence on drug release. Cassettes were immersed in $100 \mathrm{ml}$ PBS in a beaker and incubated at $37^{\circ} \mathrm{C}$ with moderate stirring. Samples were drawn at predetermined time 
intervals and the media was immediately replenished with fresh PBS. The amount of drug released was estimated spectrophotometrically as described above. The stability of fasudilloaded liposomes was evaluated for 4 weeks. For this study, liposomes $(500 \mu l)$ were stored at $4{ }^{\circ} \mathrm{C}$ and $25^{\circ} \mathrm{C}$ and samples were withdrawn on day $0,7,14,21$ and 28 and analyzed for particle size and drug content as described above. Further, liposomal stability after aerosolization was evaluated with a PennCentury Microsprayer ${ }^{\circledR}$ (Model IA-1B, PennCentury, PA). Briefly, an aliquot of liposomal sample in the form of suspension was aerosolized five times using the microsprayer device and fine droplets were collected in an Eppendorf ${ }^{\circledR}$ tube which were evaluated for vesicle size, polydispersity index and entrapment efficiency as described above.

\subsection{Uptake of liposomes by rat alveolar macrophages and pulmonary arterial smooth muscle cells}

Uptake of liposomes by macrophages was evaluated by incubating the liposomes containing FITC-Dextran with rat alveolar macrophages collected from the lungs of anesthetized male Sprague-Dawley rats (200-250 g). Briefly, the lungs were surgically removed and bronchoalveolar lavage (BAL) was performed by repeated washing of the lungs with $\mathrm{Ca}^{2+}$ and $\mathrm{Mg}^{2+}$ free Dulbecco's PBS containing $0.5 \mathrm{mM}$ disodium EDTA as described in our previously published article [17]. The resulting BAL fluid was centrifuged to obtain pellets of macrophages, which was then suspended in Hanks Balanced Salt Solution (HBSS). The cells at a density of $4 \times 10^{5}$ cells $/ \mathrm{ml}$ were then seeded onto coverslips placed in 12-well plates and incubated in a humidified chamber at $37{ }^{\circ} \mathrm{C}$ for an $\mathrm{h}$. Following incubation, an aliquot of liposomes containing FITC-Dextran suspended in HBSS was added to the cells and incubated again for an $\mathrm{h}$ at $37^{\circ} \mathrm{C}$. The cells were then fixed with acetone:methanol (1:1) at room temperature and incubated with a blocking solution containing goat serum and Tween 20 in PBS. After this, cells were incubated with monoclonal anti- $\beta$-actin primary antibodies (Sigma-Aldrich, St. Louis, MO) and Alexa Fluor ${ }^{\circledR} 594$ goat anti-mouse IgG (Invitrogen, Grand Island, NY) sequentially. Glass coverslips were placed onto glass slides and uptake of the liposomes was observed under a fluorescence microscope (IX-81, Olympus). For uptake of liposomes by rat pulmonary arterial smooth muscle cells (PASMCs, P-7), cells were seeded at a density of $5 \times 10^{3}$ cells $/ \mathrm{ml}$ and left for overnight attachment. Next day, liposomes containing FITC-Dextran were incubated with cells for an $\mathrm{h}$ at $37^{\circ} \mathrm{C}$ in a humidified chamber. Following incubation, cells were processed as described above and observed under fluorescence microscope.

\subsection{In-vivo drug absorption studies}

The pulmonary absorption of plain fasudil and fasudil-loaded liposomes were studied in adult male Sprague-Dawley rats (Charles River Laboratories, Charlotte, NC, USA) weighing between $250-300 \mathrm{~g}$. Four groups of rats $(n=6-8)$ were first anesthetized by an intramuscular (IM) injection of ketamine and xylazine cocktail $(90 \mathrm{mg} / \mathrm{kg}+10 \mathrm{mg} / \mathrm{kg})$ to receive the following treatments: plain fasudil via (i) intravenous and (ii) intratracheal routes at a dose of $10 \mathrm{mg} / \mathrm{kg}$ and, two liposomal formulations equivalent to $10 \mathrm{mg} / \mathrm{kg}$ fasudil, (iii) F-3 and (iv) F-4 administered intratracheally. Intravenous administration was performed via the penile vein and intratracheal administration was performed using a PennCentury Microsprayer ${ }^{\circledR}$ for rats [18]. Liposomal formulations were administered as dispersions in normal sterile saline. Following pulmonary or intravenous administration, blood samples were collected in citrated microcentrifuge tubes at predetermined time intervals for 12 to 24 $\mathrm{h}$. The plasma was separated by centrifugation at $5,000 \mathrm{rpm}$ for 5 minutes and stored in separate microcentrifuge tubes at $-20^{\circ} \mathrm{C}$ until further analysis. The plasma levels of fasudil were determined according to a published HPLC method [19] with little modifications using a Varian HPLC equipped with an autosampler (Varian Prostar 320, Walnut Creek, CA). The plasma sample was first deproteinized with perchloric acid and an aliquot of the supernatant 
(30 $\mu$ l) was injected into a C18 column (Inertsil $4 \mu$ ODS-3, $4.6 \times 250 \mathrm{~mm}$, GL Sciences, Inc., CA, USA). The mobile phase was an isocratic solvent system comprised of $30 \%$ acetonitrile and $70 \%$ phosphate buffer $(0.02 \mathrm{M}, \mathrm{pH} 7.4)$ and was run at a flow rate of $1 \mathrm{ml} /$ min. Fasudil eluted at $5.3 \mathrm{~min}$ and was detected by an UV detector at $320 \mathrm{~nm}$. The drug was quantified from a previously prepared standard curve using plain fasudil.

\subsection{Safety studies}

2.7.1. Cytotoxicity study-The safety of the optimized liposomal formulations (F-3 and F-4) was evaluated using an MTT assay in two different cell lines: human airway epithelial cells (Calu-3) and rat PASMCs [5]. Cells were seeded into 96-well plates at a density of $5 \times$ $10^{4}$ cells per well and incubated overnight for cells to adhere to the wells. The cells were then incubated with liposomal formulation (F-3), upon dispersion in fresh media, for $24 \mathrm{~h}$ $(100 \mu \mathrm{M} ; 100 \mu \mathrm{l}$ per well). Saline and $0.1 \%$ SDS were used as negative and positive controls, respectively. After the incubation, cells were washed with saline and then incubated for an additional $4 \mathrm{~h}$ with $100 \mu \mathrm{l}$ MTT $(0.5 \mathrm{mg} / \mathrm{ml}$ in sterile PBS). Subsequently, the medium was pipetted off and the formazan crystals were dissolved in $100 \mu \mathrm{DMSO}$ by shaking for $1 \mathrm{~h}$ on a plate shaker. The absorbance was read on a SynergyMX microplate reader (Biotek, Winnoski, VT) at $570 \mathrm{~nm}$.

2.7.2. Bronchoalveolar lavage (BAL) studies-BAL studies were performed to evaluate the safety of the optimized formulations after in vivo instillation as per our previously published procedure [20]. Male SD rats were anesthetized with ketamine/ xylazine cocktail and divided into three groups to receive following treatments intratracheally: (i) saline (negative control), (ii) fasudil liposomes (F-3), (iii) sodium dodecyl sulfate (SDS, $0.1 \%$, positive control). Twelve $\mathrm{h}$ after administration of the formulations, animals were weighed; lungs were surgically removed and weighed. The wet lung weights were reported as $\mathrm{g} / 100 \mathrm{~g}$ body weight. The lungs were then lavaged by instilling 5-ml of normal saline into the trachea and collecting the fluid after $30 \mathrm{~s}$. The BAL fluid was centrifuged at $500 \mathrm{~g}$ for $10 \mathrm{~min}$ and then the supernatant was stored at $-20{ }^{\circ} \mathrm{C}$. Protein levels in BAL fluid were assessed by Bradford assay. The enzymatic activities of lactate dehydrogenase (LDH) and alkaline phosphatase (ALP) in BAL fluid were determined by using commercial kits for assay of LDH and ALP (Pointe Scientific, Canton, MI).

\subsection{Hemodynamic studies in monocrotaline (MCT)-induced PAH rats}

The pharmacological efficacy of the formulations was studied in a rodent model of PAH. The model was developed by a single subcutaneous injection of MCT $(50 \mathrm{mg} / \mathrm{kg}$ body weight) into adult male Sprague-Dawley rats weighing between 250 and $300 \mathrm{~g}$ (Charles River Laboratories, Wilmington, MA). MCT solution was prepared by dissolving an aliquot of MCT in $0.1 \mathrm{~N} \mathrm{HCl}$ and then the $\mathrm{pH}$ was adjusted to 7.4 with $1 \mathrm{~N} \mathrm{NaOH}$. All animals were housed at TTUHSC Amarillo animal facility for 28 days with free access to food and water for developing PAH.

Four weeks later when the hypertension was established, PAH rats were anesthetized by an intramuscular injection of a cocktail of ketamine $(90 \mathrm{mg} / \mathrm{kg})$ and xylazine $(10 \mathrm{mg} / \mathrm{kg})$ and were divided into three groups $(n=8)$ to receive various treatments. Before administration of the formulations, rats were catheterized for hemodynamic measurements. For catheterization, the ventral neck area and the dorsal area between the scapulae were shaved and cleaned by scrubbing with Betadine ${ }^{\circledR}$ and ethyl alcohol. The internal jugular vein was exposed by a $2-3 \mathrm{~cm}$ incision over the right ventral neck area and kept separated using a suture. The right carotid artery was exposed and a PE-50 catheter (BD Intramedic ${ }^{\mathrm{TM}}$, Sparks, MD) was inserted 3-4 cm into the artery and secured with a suture for measurement of systemic arterial pressure. For measurement of pulmonary arterial pressure (PAP), a 
polyvinyl (PV-1, Tygon ${ }^{\circledR}$, Lima, OH) catheter was used with the top end curved at $60-65^{\circ}$ angle, threaded into the pulmonary artery via the right internal jugular vein and secured with a suture. Following catheterization, mean pulmonary arterial pressure (MPAP) and mean systemic arterial pressure (MSAP) were measured using Memscap SP844 physiological pressure transducers (Memscap AS, Scoppum, Norway) and bridge amplifiers connected to PowerLab 16/30 system with LabChart Pro 7.0 software (AD Instruments, Inc., Colorado Springs, CO). Correct positioning of the PV-1 catheter was confirmed from characteristic shapes of the pulmonary arterial pressure tracings captured by PowerLab system as reported previously [21]. Following measurement of hemodynamic parameters at zero time point, rats received one of the three treatments of fasudil (3 mg/kg): (i) plain intravenous fasudil, (ii) plain intratracheal fasudil, and (iii) liposomal formulation of fasudil (Formulation F-3) administered intratracheally. Intravenous administration was performed via the penile vein and intratracheal administration was performed using the PennCentury Microsprayer ${ }^{\circledR}$ as described above. The anesthesia was maintained throughout the procedure, and blood pressures were monitored until the pulmonary arterial pressures returned to the baseline value observed at time zero. After completion of hemodynamic measurements, the animals were sacrificed by exsanguination. All animal studies were performed in accordance with NIH Guidelines for the Care and Use of Laboratory Animals under a protocol approved by Texas Tech University Health Sciences Center (TTUHSC) Animal Care and Use Committee (AM-10012).

\subsection{Statistical Analyses}

All in vitro and in vivo data are presented as mean \pm SD and were analyzed by one-way ANOVA followed by Bonferroni post-hoc analysis using Graphpad Prism 5.0 software. Significance was defined as $p$ value $<0.05$. For pharmacokinetic analysis, standard noncompartmental analysis (Kinetica ${ }^{\circledR}$, Version 5.0, Innaphase Corp., Philadelphia, PA) was performed to calculate the area of the plasma concentration-time curve from zero to $24 \mathrm{~h}$. $\left(\mathrm{AUC}_{0 \rightarrow 24}\right)$, maximum plasma concentration $\left(\mathrm{C}_{\mathrm{max}}\right)$, and elimination half-life $\left(\mathrm{t}_{1 / 2}\right)$.

\section{RESULTS AND DISCUSSION}

\subsection{Optimization and physicochemical characterization of fasudil loaded liposomes}

Drug entrapment efficiency-Since fasudil is a weakly basic hydrophilic molecule $(\mathrm{pKa}=9.727)$, it exhibits very low entrapment efficiency in lipid-based formulations. For this reason, we first optimized the entrapment efficiency of fasudil in liposomes using passive and active loading methods and by varying drug-to-lipid ratio. Liposomes prepared by the passive method at a drug-to-lipid molar ratio of 1.5:1 showed an entrapment efficiency of $27.4 \pm 1.2 \%$ (Fig. 1A). To enhance the entrapment, we switched to active loading using $\left(\mathrm{NH}_{4}\right)_{2} \mathrm{SO}_{4}$ based transmembrane gradient method by varying $\mathrm{pH}$ of the rehydrating medium. Using this method, we prepared four sets of formulations at different $\mathrm{pH}$ values. Compared to passive loading, drug entrapment increased by 2.5 folds with $\left(\mathrm{NH}_{4}\right)_{2} \mathrm{SO}_{4}$ based transmembrane gradient method at the same drug-to-lipid molar ratio at $\mathrm{pH} 5.4$ (native) and changing the $\mathrm{pH}$ of the medium resulted in further increase in entrapment efficiency. To some extent, the data suggests that the drug entrapment by active loading method was influenced by the $\mathrm{pH}$ of the rehydrating medium and the highest increase in entrapment was observed at $\mathrm{pH} 3.0$ and 7.0. However, when the $\mathrm{pH}$ was increased to 8.0, no further increase in entrapment was observed. We further tested the influence of drug-to-lipid molar ratio on active loading of fasudil (Fig. 1B). The entrapment efficiency of fasudil was $20-25 \%$ when drug-to-lipid ratios were $0.5: 1$ and $1: 1$. When the ratio was increased to 1.5:1, a two-fold increase in loading was observed (Fig. 1B), but no appreciable increase in entrapment efficiency was observed with the increase in molar ratio 
from 1.5:1 to 2:1. Based on these data, we considered that active loading at drug-to-lipid ratio of 1.5:1 was optimum with substantial drug entrapment.

These data agree with previous studies wherein $\left(\mathrm{NH}_{4}\right)_{2} \mathrm{SO}_{4}$ based remote loading was used to increase entrapment efficiency of hydrophilic drugs. In fact, low encapsulation of hydrophilic drugs has been a limitation when small unilamellar vesicles (SUV) are to be used as drug delivery carriers [22]. Similar to fasudil, weak bases including doxorubicin and hydromorphone have been successfully encapsulated in preformed liposomes utilizing transmembrane gradient [23-26]. For example, Caelyx ${ }^{\circledR}$, a PEGylated liposomal formulation of doxorubicin, was prepared using ammonium sulfate gradient for generating indirect $\mathrm{pH}$-gradient. In this method, the presence of ammonium sulfate in the core of liposomes produces an excess availability of protons inside the vesicle because of removal of ammonium sulfate from the extraliposomal compartment resulting in a proton gradient across the liposomal membrane [27]. Unprotonated weak bases outside the vesicles can easily permeate through liposomal membranes. Once inside the vesicles, they are protonated in $\mathrm{H}^{+}$rich environment and thereby trapped in the aqueous core of the vesicles [27].

Enhanced entrapment may also result from incubation of the drug with preformed liposomes at $65^{\circ} \mathrm{C}$, which is consistent with previous assumption that unprotonated amphipathic bases are trapped within the liposomes when incubated at temperatures higher than phase transition temperatures of lipids for 30 minutes [28]. In fact, a 30-minute incubation time is reported to be optimal for maximal drug entrapment. Incubation beyond 30 minutes apparently reduces entrapment efficiency because of formation of neutral complexes between protonated fasudil and sulfate $\left(\mathrm{SO}_{4}{ }^{-2}\right)$ ions, which tend to effuse though liposomal membrane [19]. No additional entrapment was observed with the increase in $\mathrm{pH}$ from 3.0 to 7.0 or 8.0 perhaps because of reduction of proton gradient that may result from reduced protonation of $\left(\mathrm{NH}_{4}\right)_{2} \mathrm{SO}_{4}$ at $\mathrm{pH}$ as high as 7.0 or 8.0. The entrapment efficiency data suggest that ammonium sulfate based active loading is one of the most efficient methods of encapsulation of fasudil in liposomes.

However, this data raise questions regarding the influence of $\mathrm{pH}$ gradient on the entrapment efficiency. In fact, similar anomaly in entrapment efficiency was observed when Ishida et al. [19] varied $\mathrm{pH}$ and concentration of ammonium sulfate to study the influence of proton and ammonium sulfate gradient on the entrapment of fasudil into liposomes. Similar to the data presented in this manuscript, no appreciable increase in drug entrapment was observed when $\mathrm{pH}$ was varied. But the entrapment efficiency was increased when concentration of ammonium sulfate was increased. The authors theorized that $\mathrm{pH}$ gradient has perhaps a limited role in controlling entrapment of fasudil, a weakly basic drug (pKa of 9.727) [19]. Based on this published study and our data, we assume that ammonium sulfate gradient is perhaps the major player in enhancing drug loading.

Particle size and morphology-Upon efficient encapsulation of liposomes, particle size, polydispersity index, and morphology of various liposomal preparations were evaluated. The gradient produced by the presence of $\left(\mathrm{NH}_{4}\right)_{2} \mathrm{SO}_{4}$ in the vesicle core significantly influenced the size of liposomes. Compared to liposomes prepared without $\mathrm{pH}$ gradient, an overall reduction was observed in the size of liposomes prepared with $\left(\mathrm{NH}_{4}\right)_{2} \mathrm{SO}_{4}$ in combination with $\mathrm{pH}$ gradient (Fig. 1C). In the absence of transmembrane gradient, the size of liposomes was $288.7 \pm 9.9 \mathrm{~nm}$, which was decreased to $140.6 \pm 0.7 \mathrm{~nm}$ for the formulation with active loading at the $\mathrm{pH}$ of 5.4 (Fig. 1C). Such reduction in particle size can be explained by the changes in $\mathrm{pH}$ around the lipids during liposome preparation as observed by others [29, 30]. It has also been suggested that reduction in particle size results from an effect of osmotic stress produced by concentration and $\mathrm{pH}$ gradients. However, the size of liposomes prepared by $\left(\mathrm{NH}_{4}\right)_{2} \mathrm{SO}_{4}$ gradient was in the range of 100 and $250 \mathrm{~nm}$, which puts them under the category of SUVs as reported by others [31]. The SUV like size 
distribution of the liposomes suggest that hydration followed by sonication can be used for preparation of SUVs. Due to their small size, these liposomal formulations are expected to be optimal for in-vivo efficacy and avoid clearance by alveolar macrophages. Further, all liposomal formulations prepared by $\left(\mathrm{NH}_{4}\right)_{2} \mathrm{SO}_{4}$ and $\mathrm{pH}$ gradient method had a polydispersity index (PDI) in the range of 0.15 and 0.2 (Table 1), suggesting that the formulations had a homogenous size distribution. Transmission electron microscopic (TEM) image presented in Fig. 1D exhibit round or oval liposomes with a smooth surface. The formulations showed little or no aggregations indicating that the vesicles were uniformly dispersed in extraliposomal fluid. TEM has previously been used for examining the lamellar morphology, bilayer organization, and identification of possible flaws in bilayers [32]. However, the TEM photographs showed no morphological or structural changes due to incorporation of ammonium sulfate in the liposome core. Rather, we observed a uniform bed of discrete spherical vesicles that agree with vesicle size data obtained by the dynamic light scattering technique.

\subsection{In-vitro Drug Release}

The in-vitro drug release studies were performed to test the hypothesis that encapsulation of fasudil in lipidic SUVs produce sustained drug release in physiological fluid. The in-vitro release profile of the liposomal formulations obtained in PBS buffer at $37^{\circ} \mathrm{C}$ showed a continuous release of the drug for about $40 \mathrm{~h}$ (Fig. 2A). But 100\% of plain fasudil was released in the media (data not shown) suggesting that dialysis cassettes were not the rate controlling membrane for drug release. The time required to release $50 \%$ of the drug was in the range of 8 to $12 \mathrm{~h}$ for F-1, F-2, and F-5 formulations and that for F-3, and F-4 was $\sim 24 \mathrm{~h}$. During the first $24 \mathrm{~h}, \mathrm{~F}-3$ and F-4 produced $51.2 \pm 0.5 \%$ and $46.2 \pm 0.7 \%$ drug release, respectively (Fig. 2A). Since the amount of drug release was relatively small, one may question about the stability of the drug in release media. In fact, a published report suggests that fasudil is stable over an extended period of time ( $\sim 24$ weeks) at various storage conditions [19]. Further, we have used an HPLC method to quantitate fasudil in vivo which is sensitive to only intact fasudil rather than degradation products of fasudil. Thus it is unlikely that drug underwent degradation during the study period. Further, F-5 exhibited no burst release as evidenced from the negligible amount of drug released during first 30 minutes, but F-3 and F-4 formulations showed $9.1 \pm 1.4 \%$ and $11.8 \pm 2.7 \%$ drug release during the burst release phase (Fig. 2B). A slower and consistent release of fasudil from liposomal formulations suggests that drug release was due to diffusion of the drug though lipid membranes rather than disruption of the SUVs. These data agree with previous studies with liposomal fasudil [19], where carboxyfluorescien, an aqueous phase marker, was encapsulated in liposomes, and only $15 \%$ of the dye was released during a 14-day experiment [19]. In fact, the similarity among release profiles from various formulations stem from the fact that there were no differences in the type and amount of lipid used to prepare the formulations. The diffusion of the drug from the vesicles can be explained by interaction of protonated fasudil molecule with the sulfate ions in vesicle core. As discussed above, upon entering the hydrophilic core, unprotonated fasudil becomes protonated in $\mathrm{H}^{+}$ rich environment in the core and forms electrochemically neutral complexes with anionic sulfates $\left(\mathrm{SO}_{4}^{--}\right)$[33]. The resulting uncharged complex then diffuses out from the core via the lipid bilayer. Further, as the phase transition temperature for DPPC is $41^{\circ} \mathrm{C}$, which is close to the physiological temperature, $37^{\circ} \mathrm{C}$, it is reasonable to assume that the principal mechanism for drug release from liposomal formulations is diffusion of the electrochemically neutral fasudil complex through liquefied lipid membranes. This assumption is consistent with a previous study that evaluated temperature dependent phase transition of DPPC liposomal systems and showed that DPPC vesicles of $>100 \mathrm{~nm}$ undergo phase transition at around $37-39^{\circ} \mathrm{C}$ [34]. Further, encapsulation of small molecules such as fasudil is unlikely to alter phase transition properties of lipid vesicles at high drug to lipid 
ratios. Overall, the in-vitro release data suggest that F-3 and F-4 formulations would be efficacious in delivering fasudil in the distal pulmonary arterioles for a prolonged period of time and would likely provide sustained vasodilation.

\subsection{In-vitro Stability Studies}

Reduction in drug entrapment due to drug leakage and aggregation of vesicles are major concerns with the stability of liposomal formulations. To examine the stability of liposomal formulations, we assessed the changes in particle size and drug entrapment of F-3 and F-4 formulations which showed maximal loading. The formulations were stored at $4{ }^{\circ} \mathrm{C}$ and $25^{\circ} \mathrm{C}$, and the particle size and drug entrapments were monitored for 4 weeks. No changes in vesicle size was observed in formulations stored at $4^{\circ} \mathrm{C}$ and $25^{\circ} \mathrm{C}$, suggesting that the vesicles remained well separated from each other and formed no aggregates during the storage period (Fig. 3A). Therefore, no change in particle size can be attributed to the stability provided by the optimum zeta potential which prevents the particles from coalescing and aggregating. When stored at $4{ }^{\circ} \mathrm{C}$, no change in drug entrapment was observed for 21 days, but a $10-15 \%$ reduction in drug entrapment was observed upon storage for 28 days (Fig. 3B). However, storage at room temperature $\left(25^{\circ} \mathrm{C}\right)$ resulted in about $30-35 \%$ drug loss over 28 days, which is $2-3$ times higher than drug loss that occurred upon storage at $4^{\circ} \mathrm{C}$. Moreover the lipids, cholesterol and phospholipid, used to prepare liposomes are unlikely to undergo degradation during storage at $4^{\circ} \mathrm{C}$ [27] and it has also been shown that liposomal formulations can release drugs from 2 weeks to as long as several months [35], indicating that the integrity of liposomes is unlikely to be adversely affected in the physiological fluid.

In addition to studying the stability at various storage temperatures, we also studied stability upon aerosolization. In fact, aerosolization breaks larger droplets into smaller ones and delivers the formulations in the form of fine mists. But the force applied for nebulization may rupture the liposomes and release the drug prematurely. This may also result in leakage of drugs from some liposomes. To test this, we nebulized the formulation 5 times to mimic intratracheal administration into rats and evaluated various physicochemical properties of liposomes. The data presented in Table 2 show that there were no significant differences in vesicle size, polydispersity index and entrapment efficiency before and after nebulization of the liposomes, suggesting that liposomal integrity was not affected due to the force applied to administer the formulations using PennCentury Microsprayers ${ }^{\circledR}$.

\subsection{Uptake of liposomes by rat alveolar macrophages and PASMCs}

Alveolar macrophages, present on lung epithelial surfaces and responsible for engulfing and clearing of inhaled particles, may influence the clearance of liposomal formulations. Further, previously published reports suggest that particles less than $250 \mathrm{~nm}$ can escape clearance by macrophages [36]. Microscopic images suggest little or no uptake of liposomes by macrophages (Fig. 4A), indicating that liposomes were not cleared via macrophageal uptake mechanism. Particles are intended to produce their therapeutic response in smooth muscle cells by inhibiting Rho-kinase and hence reduce mean pulmonary arterial pressure. To demonstrate this, we performed another set of experiment using rat PASMCs and observed that there was significant uptake of liposomes by these cells (Fig. 4B). Superimposed images of FITC (green) and TRITC (red) channels show the localization, as visualized by yellow fluorescence, indicating that liposomal formulations can act on vascular smooth muscles and produce therapeutic effect of the drug. Further transport studies using cells of airway epithelium are required to track the movement of fasudil or liposomes across the pulmonary vasculature. 


\subsection{In-vivo Absorption Studies}

In pulmonary absorption studies, the pharmacokinetics of two formulations with maximal drug loading (F-3 and F-4) were compared with that of plain fasudil administered via intravenous or pulmonary routes (Fig. 5). Intravenous administration of fasudil resulted in a quick rise in drug plasma concentrations with a $C_{\max }$ of $298 \pm 10 \mathrm{ng} / \mathrm{ml}$ followed by a rapid decline (Fig. 5A), whereas pulmonary administration of plain fasudil resulted in an appreciable increase in plasma concentrations $\left(\mathrm{C}_{\max }=66.2 \pm 13.6 \mathrm{ng} / \mathrm{ml}\right.$, Fig. 5A). Unlike intravenous route, the elimination of fasudil administered by pulmonary route was slow (Fig. 5A and Table 3), which is evident from the differences in elimination half-lives of fasudil administered by the two routes. The elimination half-life $\left(t_{1 / 2}\right)$ of fasudil administered by pulmonary route $(1.17 \pm 0.21 \mathrm{~h}$.) was significantly higher than that of fasudil given intravenously $\left(\mathrm{t}_{1 / 2}=0.39 \pm 0.12 \mathrm{~h}\right.$.) $(p<0.05)$ (Fig. 5A, Table 3$)$. The relative bioavailability of fasudil after pulmonary administration was $\approx 40 \%$ (Table 3 ). However, the pharmacokinetic profiles observed after intratracheal administration of two liposomal formulations (F-3 and F-4) were completely different from those observed with plain intratracheal and intravenous fasudil (Fig. 5B). F-3 formulation showed a $C_{\max }$ of $89.4 \pm 29.7$ $\mathrm{ng} / \mathrm{ml}$ at $8 \mathrm{~h}$. followed by a sharp decline in plasma fasudil levels with no detectable concentrations at $18 \mathrm{~h}$. Similarly, F-4 formulation produced a $C_{\max }$ of $86.9 \pm 11.9 \mathrm{ng} / \mathrm{ml}$ at 2 $\mathrm{h}$. Pharmacokinetic analysis of the in-vivo data revealed that fasudil liposomes resulted in a remarkable extension of elimination $\mathrm{t}_{1 / 2}(4.71 \pm 0.72 \mathrm{~h}$. for F-3 and $3.44 \pm 0.49 \mathrm{~h}$. for F- 4$)$. In fact, $\mathrm{t}_{1 / 2}$ of both formulations was $\sim 3-4$ and 10 times, respectively longer than plain fasudil after pulmonary and intravenous administration. The $\mathrm{IC}_{50}$ of fasudil against Rho-kinase is reported to be between 1.9-3.7 $\mu \mathrm{M}[37,38]$. Based on the published data we assume that the plasma concentrations of fasudil were above therapeutic levels for at least $15 \mathrm{~h}$ with liposomal formulations, which can be translated into a once-a-day or twice-a-day dosing regimen. Similar to in vitro data, in-vivo absorption profiles also show a trend of extended release although the two profiles were not similar. Differences between in vitro release and in vivo absorption profiles can be attributed to a number of factors including mechanisms of absorption, distribution and elimination of liposomes administered via the pulmonary, physicochemical characteristics of the aerosolized liposomes, macrophage dependent clearance, lipid degrading enzymes, and transport of intact liposomes from lungs to the systemic circulation, metabolic degradation and protein binding. Further, sustained release behavior of liposomes may also result from multiple factors. First, upon inhalation, liposomes may act as reservoirs that remain submerged in the respiratory fluid and release the drug continuously. In fact, particulate carriers with particle size below $1 \mu \mathrm{m}$ can avoid phagocytosis by alveolar macrophages $[39,40]$ and thereby perhaps form aggregates in the respiratory mucosa and act like reservoirs or depot. Based on this assumption, the drug is expected to be released from the liposomes by diffusion though lipid bilayer. Release of the drug due to rupture of liposomal bilayer is unlikely since lipids used are resistant to degradation by enzymes present in respiratory mucosa [41, 42]. However, dissolution of liposomes in the surfactant rich respiratory fluid cannot be ruled out. A fraction of the drug is likely to be released due to dissolution of liposomes in the respiratory mucosa. In either case, drug released from liposomes will traverse the blood airway barrier and enter arterioles via the adventitial side to produce its vasodilatory effects. A second mechanism for sustained release of the drug may involve transport of intact liposomes or released drug to the systemic circulation via the air-blood barrier. Upon entering the systemic circulation fasudil may diffuse out of the liposomes to the systemic circulation. Fasudil in the circulating blood enters the smooth muscles of the pulmonary arterioles from the endothelial side to produce its therapeutic effect. Further studies using ex-vivo models such as isolated perfused lungs are required to assess the relative contribution of above described absorptive mechanisms upon intratracheal administration of liposomes. However, one of the important limitations of this in vivo study is that it was performed in healthy animals. Since PAH 
animals undergo several pathological changes, pharmacokinetics of the drug in PAH rats could be slightly different from those observed in healthy animals. Future studies should delineate the differences between the pharmacokinetics of the drug in healthy and diseased animals.

Safety Studies-In-vitro cytotoxicity studies were performed to determine the effects of liposomal formulations on two different cell lines: Calu-3 and rat PASMCs. Cells were treated with saline (negative control) and $0.1 \% \mathrm{w} / \mathrm{v}$ sodium dodecyl sulfate (SDS) (positive control) showed cell viability of $99 \%$ and $15 \%$, respectively (Fig. 6A). When Calu-3 cells were treated with $100 \mu \mathrm{M} \mathrm{F}-3$ formulations for $24 \mathrm{~h}$, the cell viability was $86.19 \pm 13.66 \%$ (Fig. 6A). One primary cell line (rat PASMCs) treated with $100 \mu \mathrm{M}$ of liposomes for $24 \mathrm{~h}$ showed similar cell viability. In none of the cases was cell viability below $70 \%$, suggesting that formulations were toxic neither to immortalized Calu-3 cells nor to primary PASMCs. These observations were expected since lipids used to prepare liposomes have long been known to produce little or no cytotoxicity.

To further evaluate the safety, BAL was performed after taking weights of wet lungs as discussed above in the method section. The weight of wet lungs, expressed in lung weight per $100 \mathrm{~g}$ of body weight (L/B), of saline treated animals was $0.397 \pm 0.0047$ and that for SDS treated animals was $0.614 \pm 0.0368$ (Fig. 6B), suggesting formation of edema due to accumulation of extracellular fluid into the epithelial cells of the respiratory wall in SDS treated lungs. For liposomal formulation containing fasudil (F-3), L/B ratio was $0.42 \pm 0.0116$, indicating no substantial lung injury or edema formation. The levels of two injury markers, LDH and ALP, as well as total protein concentration in the BAL collected from animals treated with formulation F-3 were not significantly different from that observed in the lungs of animals that received saline (Fig. 6C and 6D) which were significantly higher in SDS treated animals. Overall, data presented in Fig. 6 establish the safety profile of liposome based formulations after single administration, but long-term safety profiles of fasudil formulations after multiple administrations should be evaluated.

\subsection{Efficacy of Liposomal Fasudil in PAH Rats}

The pharmacological efficacy of the formulations in reducing mean pulmonary arterial pressure (MPAP) was studied in an MCT induced rat model of PAH. The signs induced by a single subcutaneous injection of MCT $(50-60 \mathrm{mg} / \mathrm{kg}$ ) resemble those of clinical PAH that include increased MPAP, right ventricular hypertrophy, pulmonary vascular remodeling, and reduced luminal diameter of small pulmonary arterioles [43, 44]. In this study, the average MPAP was $38.0 \pm 9.4 \mathrm{~mm} \mathrm{Hg} 4$ weeks after MCT injection as compared to $15.6 \pm 4.4$ $\mathrm{mm} \mathrm{Hg}$ in sham animals. In agreement with earlier reports [14, 45, 46], intravenous administration of plain fasudil $(3 \mathrm{mg} / \mathrm{kg}$ ) produced a $45.6 \pm 17.0 \%$ decrease in MPAP, but its vasodilatory effect quickly subsided within 60 to 80 minutes (Fig. 7A). Intratracheal administration of plain fasudil also caused a $38.3 \pm 7.3 \%$ decrease in MPAP, although the duration of the vasodilatory effect was slightly longer than that produced by intravenous fasudil (Fig. 7A and 7C). However, when liposomal fasudil (Formulation F-3) was administered via the pulmonary route, the duration of vasodilatory effect was extended up to 200 minutes (Fig. 7A). The maximal reduction in MPAP produced by liposomal fasudil was $37.6 \pm 5.7 \%$ which was similar to that produced by plain fasudil administered via either route. However, unlike plain fasudil administered intravenously or intratracheally, liposomal fasudil continued to produce pulmonary vasodilation even $3.5 \mathrm{~h}$ post instillation, i.e., a $20.0 \pm 8.7 \%$ reduction in MPAP at 200 minutes (Fig. 7A). The data concerning the pharmacological efficacy presented in Fig. 7 reflects the absorption profile of the formulation presented in Fig. 5, wherein the $t_{1 / 2}$ of liposomal fasudil was significantly longer than that of plain fasudil. To calculate the duration of pulmonary vasodilatory effects, 
we used 15\% reduction in MPAP as baseline and plotted the values to compare the duration of three treatments. This baseline was chosen based on previous studies that show a 15 to $20 \%$ reduction in MPAP is therapeutically relevant and significant [47]. The liposomal fasudil maintained pulmonary vasodilatory effects for $3 \mathrm{~h}$. (172 \pm 17 minutes) while intravenous and pulmonary plain fasudil caused vasodilation for $50.0 \pm 10.4$ and $60.0 \pm 15.3$ minutes, respectively (Fig. 7C). These data further confirm that liposomal fasudil produced a prolonged vasodilation that would offer therapeutic advantages over the plain drug. However, concerns may be raised regarding the candidacy of fasudil as a drug for development into controlled formulations. Since the effective dose in animal was $3 \mathrm{mg} / \mathrm{kg}$, one might argue that a much larger dose (200-300 mg) would be required in humans and thus the proposed formulation would be not be viable for use in human patients. But calculation of human dose based on the allometric [48] and body surface area principles [49] suggest that $3 \mathrm{mg} / \mathrm{kg}$ dose in rats is equivalent to $\sim 35-40 \mathrm{mg}$ in 70-kg human subjects. In fact, fasudil used at a dose of 30 or $40 \mathrm{mg}$ was found to effective in reducing MPAP in PAH patients $[45,50]$. Thus this study provides convincing data in support of an inhaled controlled release formulation of fasudil for the treatment of PAH.

To evaluate the pulmonary selectivity of the formulation, we also measured the mean systemic arterial pressure (MSAP) and calculated the reduction in MSAP produced by the formulations (Fig. 7B). Intravenous and intratracheal fasudil produced a $45.6 \pm 8.4 \%$ and $38.1 \pm 10.8 \%$ reduction in MSAP, respectively (Fig. 7B). The extent of reduction of MSAP produced by plain fasudil was close to that observed in MPAP (Fig. 7A), suggesting that fasudil when given intravenously does not exhibit much selectivity toward the pulmonary circulation and thus produces peripheral vasodilation. However, intratracheal liposomal fasudil produced a $26.4 \pm 7.4 \%$ reduction in MSAP, which was significantly less $(p<0.05)$ than that produced by intravenous and intratracheal plain fasudil. A point-by-point comparison of hemodynamic data presented in Fig. 7B also suggests that the reduction in systemic arterial pressure produced by aerosolized liposomal fasudil was consistently lower than that produced by plain fasudil administered either by intravenous or pulmonary route. In addition to pulmonary selectivity, fasudil encapsulated in liposomes is likely to reduce the dosing frequency and minimize the fluctuations in MSAP due to multiple injections or continuous infusion that leads to serious side effects including syncope and cardiovascular collapse [51]. Overall, the hemodynamic study suggests that intratracheal instillation of fasudil encapsulated in liposomes was therapeutically active and the drug was released from liposomes over time. There was a continuous influx of fasudil into the pulmonary arteries that provided sustained vasodilation with reduced effect on systemic arterial pressure than that of plain fasudil. These data are in agreement with in-vivo absorption profile wherein a continuous absorption of fasudil was observed. However, the in vivo efficacy study was limited to evaluation of only one of the pharmacological parameters, mean pulmonary arterial pressures. Evaluation of other parameters such as influence of the formulations in reducing vascular resistance and ameliorating other pathological changes in PAH would have provided more useful information regarding the therapeutic benefit of liposomal fasudil. Future studies will be directed toward assessing various cellular and vascular markers upon administration of the formulations.

\section{CONCLUSIONS}

In summary, this is the first study to investigate the feasibility of an aerosolized controlled release formulation of fasudil that produces a sustained pulmonary preferential vasodilation and ameliorates the severity of MCT-induced PAH. Liposomal formulations can be used as vehicles for pulmonary delivery of fasudil as demonstrated by favorable physicochemical properties. The drug exhibited an extended $\mathrm{t}_{1 / 2}$ following in-vivo administration in rats. Compared to intravenous fasudil, a single dose of the optimized liposomal formulations 
produced sustained pulmonary vasodilation for $\sim 3 \mathrm{~h}$ and produced minimal impact on MSAP. Nanosized liposomes were safe after intratracheal delivery, escaped clearance by alveolar macrophages and were internalized by PASMCs. Thus, the data presented suggest that aerosolized liposomes of fasudil can be used for sustained and pulmonary preferential vasodilation at a reduced dosing frequency and can potentially be used as a viable anti-PAH therapy. However, further studies are required to establish the long-term efficacy of fasudil liposomes in providing protection against PAH related lesions.

\section{Acknowledgments}

The authors sincerely acknowledge Mr. Charles Linch at the Department of Medical Photography and Electron Microscopy of Texas Tech University Health Sciences Center, Lubbock, TX, for his help with the transmission electron-microscopy (TEM) experiments. This work was supported in part by an American Recovery and Reinvestment Act Fund, NIH 1R15HL103431 to Dr. Fakhrul Ahsan. Primary PASM cell lines were developed with a financial support from Cardiovascular and Pulmonary Research, University of Colorado, Denver and NIH-PPG \# 2P01HL014985-36A1 (Adaptations to Hypoxia) to Drs. Eva Nozik-Grayck and Kurt Stenmark.

\section{REFERENCES}

1. Corris PA. Alternatives to lung transplantation: treatment of pulmonary arterial hypertension. Clin Chest Med. 2011; 32:399-410. [PubMed: 21511098]

2. Vachiery JL. Prostacyclins in pulmonary arterial hypertension: the need for earlier therapy. Adv Ther. 2011; 28:251-269. [PubMed: 21455725]

3. Chen L, Nakano K, Kimura S, Matoba T, Iwata E, Miyagawa M, Tsujimoto H, Nagaoka K, Kishimoto J, Sunagawa K, Egashira K. Nanoparticle-mediated delivery of pitavastatin into lungs ameliorates the development and induces regression of monocrotaline-induced pulmonary artery hypertension. Hypertension. 2011; 57:343-350. [PubMed: 21220711]

4. Kimura S, Egashira K, Chen L, Nakano K, Iwata E, Miyagawa M, Tsujimoto H, Hara K, Morishita R, Sueishi K, Tominaga R, Sunagawa K. Nanoparticle-mediated delivery of nuclear factor kappaB decoy into lungs ameliorates monocrotaline-induced pulmonary arterial hypertension. Hypertension. 2009; 53:877-883. [PubMed: 19307469]

5. Gupta V, Rawat A, Ahsan F. Feasibility study of aerosolized prostaglandin E1 microspheres as a noninvasive therapy for pulmonary arterial hypertension. J Pharm Sci. 2010; 99:1774-1789. [PubMed: 19894275]

6. Bailey MM, Berkland CJ. Nanoparticle formulations in pulmonary drug delivery. Med Res Rev. 2009; 29:196-212. [PubMed: 18958847]

7. Taylor KM, Newton JM. Liposomes for controlled delivery of drugs to the lung. Thorax. 1992; 47:257-259. [PubMed: 1585287]

8. Bhavane R, Karathanasis E, Annapragada AV. Agglomerated vesicle technology: a new class of particles for controlled and modulated pulmonary drug delivery. J Control Release. 2003; 93:15-28. [PubMed: 14602418]

9. Ulrich AS. Biophysical aspects of using liposomes as delivery vehicles. Biosci Rep. 2002; 22:129150. [PubMed: 12428898]

10. Lopez-Berestein G. Liposomes as carriers of antimicrobial agents. Antimicrob Agents Chemother. 1987; 31:675-678. [PubMed: 3300535]

11. Hajos F, Stark B, Hensler S, Prassl R, Mosgoeller W. Inhalable liposomal formulation for vasoactive intestinal peptide. Int J Pharm. 2008; 357:286-294. [PubMed: 18328650]

12. Kleemann E, Schmehl T, Gessler T, Bakowsky U, Kissel T, Seeger W. Iloprost-containing liposomes for aerosol application in pulmonary arterial hypertension: formulation aspects and stability. Pharm Res. 2007; 24:277-287. [PubMed: 17211729]

13. Stark B, Debbage P, Andreae F, Mosgoeller W, Prassl R. Association of vasoactive intestinal peptide with polymer-grafted liposomes: structural aspects for pulmonary delivery. Biochim Biophys Acta. 2007; 1768:705-714. [PubMed: 17204237]

14. Oka M, Fagan KA, Jones PL, McMurtry IF. Therapeutic potential of RhoA/Rho kinase inhibitors in pulmonary hypertension. Br J Pharmacol. 2008; 155:444-454. [PubMed: 18536743] 
15. Shibuya M, Hirai S, Seto M, Satoh S, Ohtomo E. Effects of fasudil in acute ischemic stroke: results of a prospective placebo-controlled double-blind trial. J Neurol Sci. 2005; 238:31-39. [PubMed: 16005902]

16. Zhang JA, Anyarambhatla G, Ma L, Ugwu S, Xuan T, Sardone T, Ahmad I. Development and characterization of a novel Cremophor EL free liposome-based paclitaxel (LEP-ETU) formulation. Eur J Pharm Biopharm. 2005; 59:177-187. [PubMed: 15567316]

17. Patel B, Gupta V, Ahsan F. PEG-PLGA based large porous particles for pulmonary delivery of a highly soluble drug, low molecular weight heparin. J Control Release. 2012; 162:310-320. [PubMed: 22800582]

18. Bai S, Ahsan F. Inhalable liposomes of low molecular weight heparin for the treatment of venous thromboembolism. J Pharm Sci. 2010; 99:4554-4564. [PubMed: 20845454]

19. Ishida T, Takanashi Y, Doi H, Yamamoto I, Kiwada H. Encapsulation of an antivasospastic drug, fasudil, into liposomes, and in vitro stability of the fasudil-loaded liposomes. Int J Pharm. 2002; 232:59-67. [PubMed: 11790490]

20. Gupta V, Davis M, Hope-Weeks LJ, Ahsan F. PLGA microparticles encapsulating prostaglandin E1-hydroxypropyl-beta-cyclodextrin (PGE1-HPbetaCD) complex for the treatment of pulmonary arterial hypertension (PAH). Pharm Res. 2011; 28:1733-1749. [PubMed: 21626061]

21. Crossno JT Jr, Garat CV, Reusch JE, Morris KG, Dempsey EC, McMurtry IF, Stenmark KR, Klemm DJ. Rosiglitazone attenuates hypoxia-induced pulmonary arterial remodeling. Am J Physiol Lung Cell Mol Physiol. 2007; 292:L885-L897. [PubMed: 17189321]

22. Kulkarni SB, Betageri GV, Singh M. Factors affecting microencapsulation of drugs in liposomes. J Microencapsul. 1995; 12:229-246. [PubMed: 7650588]

23. Lewrick F, Suss R. Remote loading of anthracyclines into liposomes. Methods Mol Biol. 2010; 605:139-145. [PubMed: 20072878]

24. Mayer LD, Bally MB, Cullis PR. Uptake of adriamycin into large unilamellar vesicles in response to a $\mathrm{pH}$ gradient. Biochim Biophys Acta. 1986; 857:123-126. [PubMed: 3964703]

25. Ong JC, Sun F, Chan E. Development of stealth liposome coencapsulating doxorubicin and fluoxetine. J Liposome Res. 2011

26. Tu S, McGinnis T, Krugner-Higby L, Heath TD. A mathematical relationship for hydromorphone loading into liposomes with trans-membrane ammonium sulfate gradients. J Pharm Sci. 2010; 99:2672-2680. [PubMed: 20014429]

27. Haran G, Cohen R, Bar LK, Barenholz Y. Transmembrane ammonium sulfate gradients in liposomes produce efficient and stable entrapment of amphipathic weak bases. Biochim Biophys Acta. 1993; 1151:201-215. [PubMed: 8373796]

28. Uster PS, Allen TM, Daniel BE, Mendez CJ, Newman MS, Zhu GZ. Insertion of poly(ethylene glycol) derivatized phospholipid into pre-formed liposomes results in prolonged in vivo circulation time. FEBS Lett. 1996; 386:243-246. [PubMed: 8647291]

29. Dos Santos N, Cox KA, McKenzie CA, van Baarda F, Gallagher RC, Karlsson G, Edwards K, Mayer LD, Allen C, Bally MB. pH gradient loading of anthracyclines into cholesterol-free liposomes: enhancing drug loading rates through use of ethanol. Biochim Biophys Acta. 2004; 1661:47-60. [PubMed: 14967474]

30. Ertel A, Marangoni AG, Marsh J, Hallett FR, Wood JM. Mechanical properties of vesicles. I. Coordinated analysis of osmotic swelling and lysis. Biophys J. 1993; 64:426-434. [PubMed: 8457668]

31. Bunuales M, Duzgunes N, Zalba S, Garrido MJ, de Ilarduya CT. Efficient gene delivery by EGFlipoplexes in vitro and in vivo. Nanomedicine. 2011; 6:89-98. [PubMed: 21182421]

32. Zasadzinski JA. Transmission electron microscopy observations of sonication-induced changes in liposome structure. Biophys J. 1986; 49:1119-1130. [PubMed: 3719073]

33. Ishida T, Takanashi Y, Kiwada H. Safe and efficient drug delivery system with liposomes for intrathecal application of an antivasospastic drug, fasudil. Biol Pharm Bull. 2006; 29:397-402. [PubMed: 16508135]

34. R.L. Biltonen DL. The use of differential scanning calorimetry as a tool to characterize liposome preparations. Chem Phys Lipids. 1993; 64:129-142. 
35. Lindner LH, Hossann M. Factors affecting drug release from liposomes. Curr Opin Drug Discov Devel. 2010; 13:111-123.

36. Chono S, Tanino T, Seki T, Morimoto K. Uptake characteristics of liposomes by rat alveolar macrophages: influence of particle size and surface mannose modification. J Pharm Pharmacol. 2007; 59:75-80. [PubMed: 17227623]

37. Masumoto A, Mohri M, Shimokawa H, Urakami L, Usui M, Takeshita A. Suppression of coronary artery spasm by the Rho-kinase inhibitor fasudil in patients with vasospastic angina. Circulation. 2002; 105:1545-1547. [PubMed: 11927519]

38. Shimokawa H, Seto M, Katsumata N, Amano M, Kozai T, Yamawaki T, Kuwata K, Kandabashi T, Egashira K, Ikegaki I, Asano T, Kaibuchi K, Takeshita A. Rho-kinase-mediated pathway induces enhanced myosin light chain phosphorylations in a swine model of coronary artery spasm. Cardiovasc Res. 1999; 43:1029-1039. [PubMed: 10615430]

39. Champion JA, Walker A, Mitragotri S. Role of particle size in phagocytosis of polymeric microspheres. Pharm Res. 2008; 25:1815-1821. [PubMed: 18373181]

40. Makino K, Yamamoto N, Higuchi K, Harada N, Ohshima H, Terada H. Phagocytic uptake of polystyrene microspheres by alveolar macrophages: effects of the size and surface properties of the microspheres. Colloids Surf B Biointerfaces. 2003; 27:33-39.

41. Duncan JE, Hatch GM, Belik J. Susceptibility of exogenous surfactant to phospholipase A2 degradation. Canad J Physiol Pharmacol. 1996; 74:957-963. [PubMed: 8960386]

42. Dizon-Co L, Ikegami M, Ueda T, Jobe AH, Lin WH, Turcotte JG, Notter RH, Rider ED. In vivo function of surfactants containing phosphatidylcholine analogs. Am J Resp Crit Care Med. 1994; 150:918-923. [PubMed: 7921463]

43. Todd L, Mullen M, Olley PM, Rabinovitch M. Pulmonary toxicity of monocrotaline differs at critical periods of lung development. Pediatr Res. 1985; 19:731-737. [PubMed: 3161000]

44. van Suylen RJ, Smits JF, Daemen MJ. Pulmonary artery remodeling differs in hypoxia- and monocrotaline-induced pulmonary hypertension. Am J Resp Crit Care Med. 1998; 157:14231428. [PubMed: 9603118]

45. Fukumoto Y, Matoba T, Ito A, Tanaka H, Kishi T, Hayashidani S, Abe K, Takeshita A, Shimokawa H. Acute vasodilator effects of a Rho-kinase inhibitor, fasudil, in patients with severe pulmonary hypertension. Heart. 2005; 91:391-392. [PubMed: 15710736]

46. Nagaoka T, Fagan KA, Gebb SA, Morris KG, Suzuki T, Shimokawa H, McMurtry IF, Oka M. Inhaled Rho kinase inhibitors are potent and selective vasodilators in rat pulmonary hypertension. Am J Resp Crit Care Med. 2005; 171:494-499. [PubMed: 15563635]

47. Jiang BH, Tawara S, Abe K, Takaki A, Fukumoto Y, Shimokawa H. Acute vasodilator effect of fasudil, a Rho-kinase inhibitor, in monocrotaline-induced pulmonary hypertension in rats. $\mathbf{J}$ Cardiovasc Pharmacol. 2007; 49:85-89. [PubMed: 17312448]

48. Mahmood I. Application of allometric principles for the prediction of pharmacokinetics in human and veterinary drug development. Adv Drug Deliv Rev. 2007; 59:1177-1192. [PubMed: 17826864]

49. Center for Drug Evaluation and Research. Rockville, MD: Food and Drug Administration; 2005. Guidance for Industry: Estimating the Maximum safe Starting Dose in Initial Clinical Trials for Therapeutics in Adult Healthy Volunteers; p. 1-27.

50. Fujita H, Fukumoto Y, Saji K, Sugimura K, Demachi J, Nawata J, Shimokawa H. Acute vasodilator effects of inhaled fasudil, a specific Rho-kinase inhibitor, in patients with pulmonary arterial hypertension. Heart Vessels. 2010; 25:144-149. [PubMed: 20339976]

51. Gupta V, Ahsan F. Inhalational therapy for pulmonary arterial hypertension: current status and future prospects. Crit Rev Ther Drug Carrier Syst. 2010; 27:313-370. [PubMed: 20932241] 

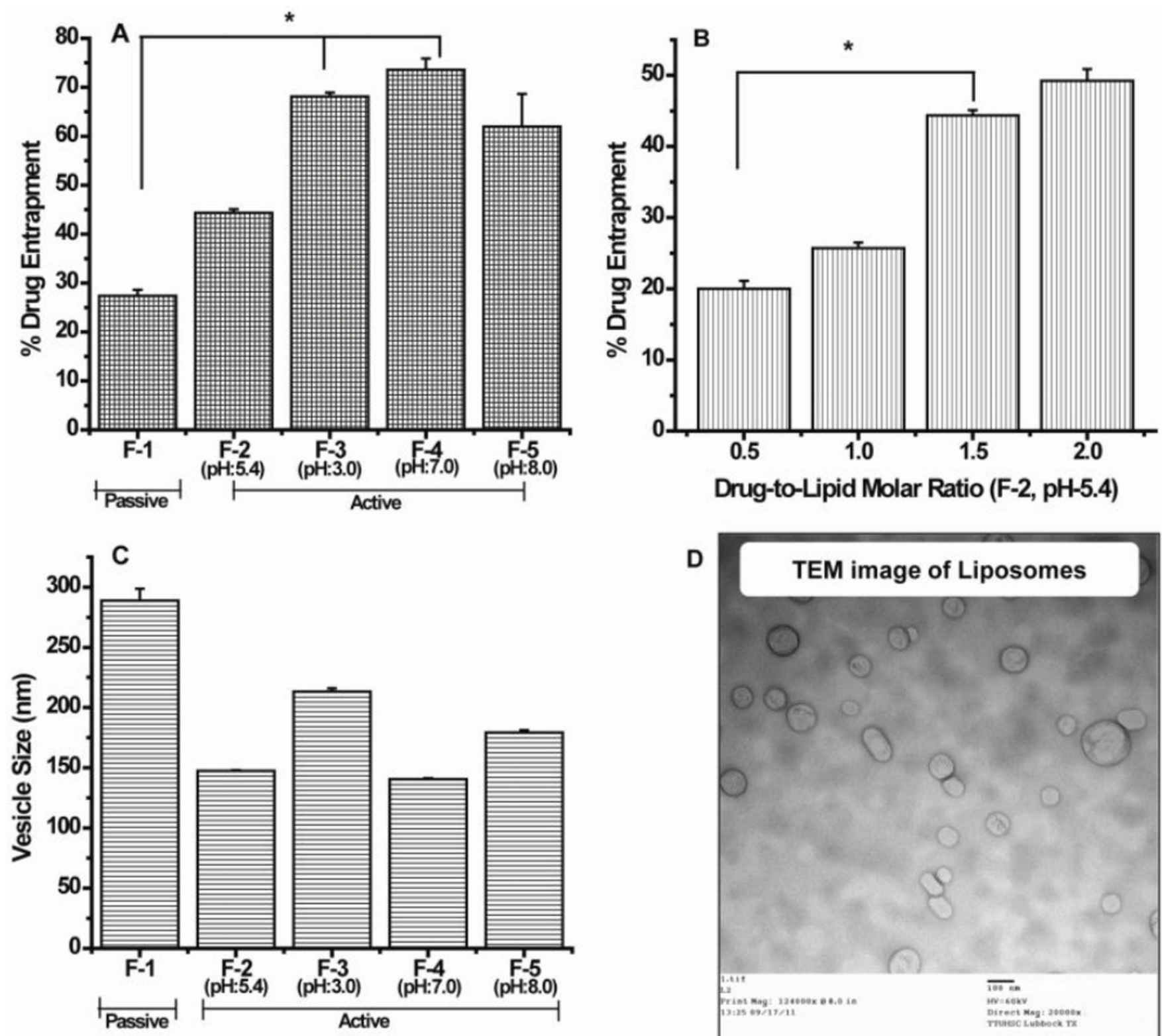

D

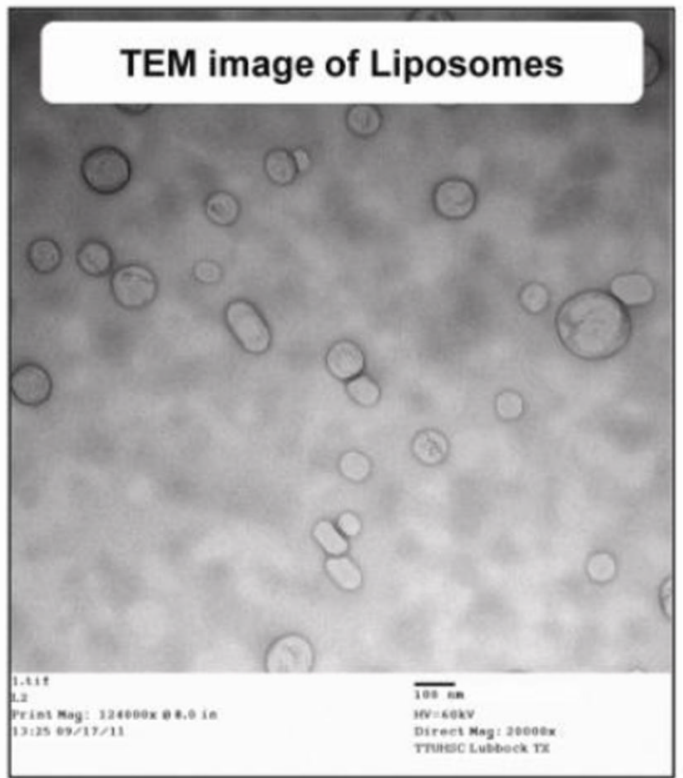

Figure 1.

(A) \% Drug entrapment upon passive and active loading at a drug-to-lipid molar ratio of 1.5:1, (B) Influence of drug-to-lipid molar ratio on entrapment efficiency upon active loading, (C) vesicle size of liposomes, and (D) respresentative transmission electron microscopic image of fasudil loaded liposomes. Data represent mean \pm standard deviation (n $=3$ ). ${ }^{*}$ Results are significantly different $(p<0.05)$, (A) F-3 and F-4 compared with F-1 and (B) molar ratio 1.5 compared with 0.5 . 

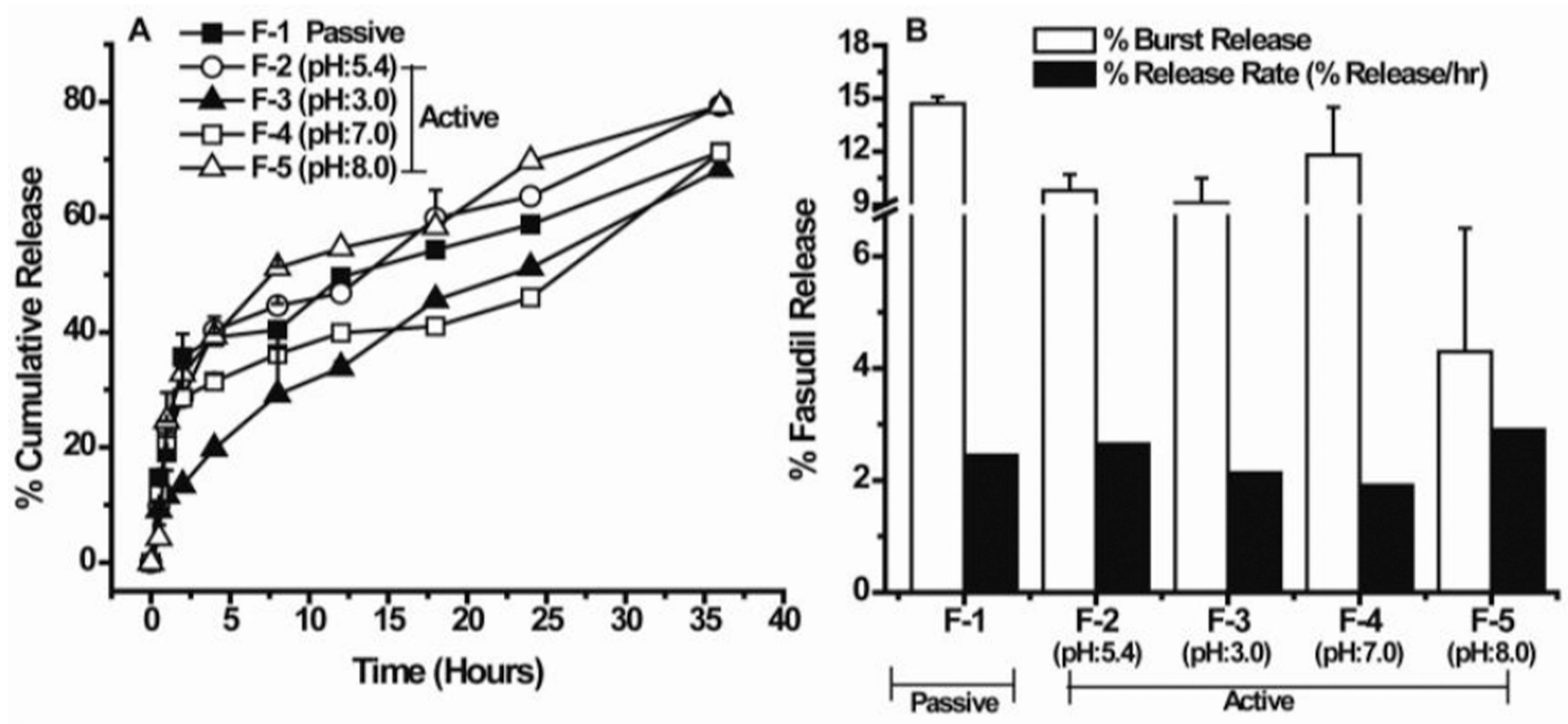

Figure 2.

In-vitro release profiles of fasudil-loaded liposomal formulations in PBS (pH 7.4) at $37^{\circ} \mathrm{C}$ : (A) Release profiles of liposomes F-1 through F-5, and (B) Release kinetics showing burst release (release in first 30 minutes) and $\%$ release rate/hr. Data represent mean \pm standard deviation $(\mathrm{n}=3)$. 

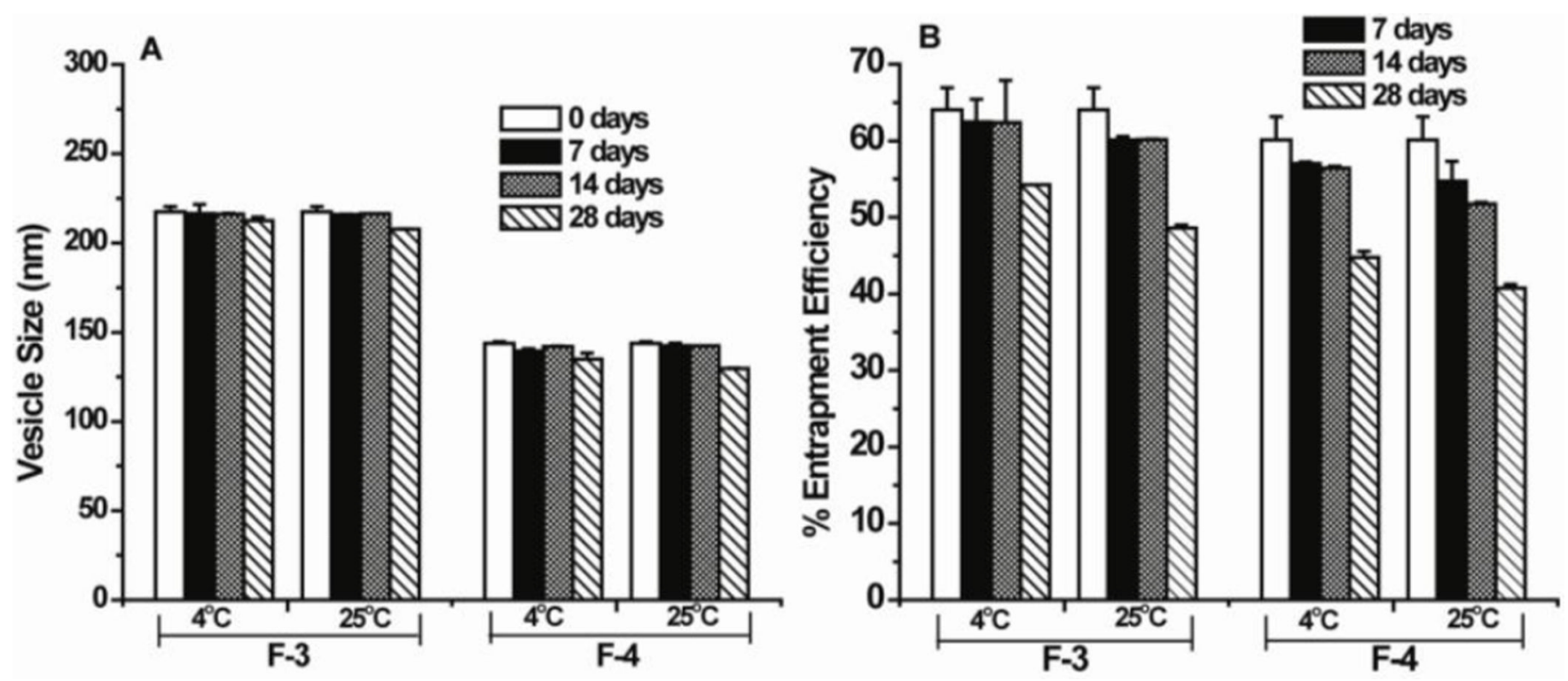

Figure 3.

Influence of storage temperature and length of storage on (A) particle size and (B) drug entrapment efficiency Formulations were stored at $4{ }^{\circ} \mathrm{C}$ and $25^{\circ} \mathrm{C}$ over a period of 4 weeks. Data represent mean \pm standard deviation $(n=3)$. 
A

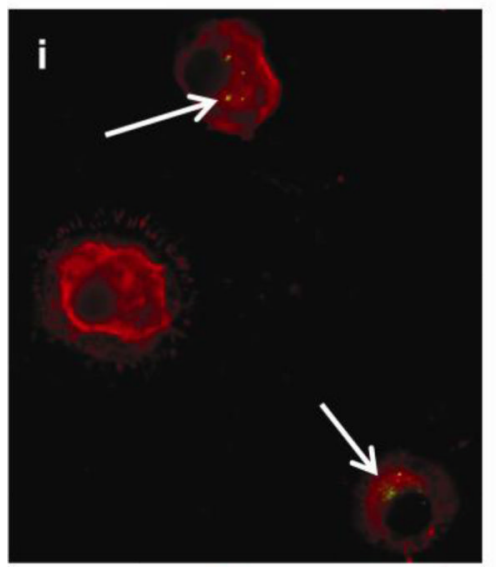

ii
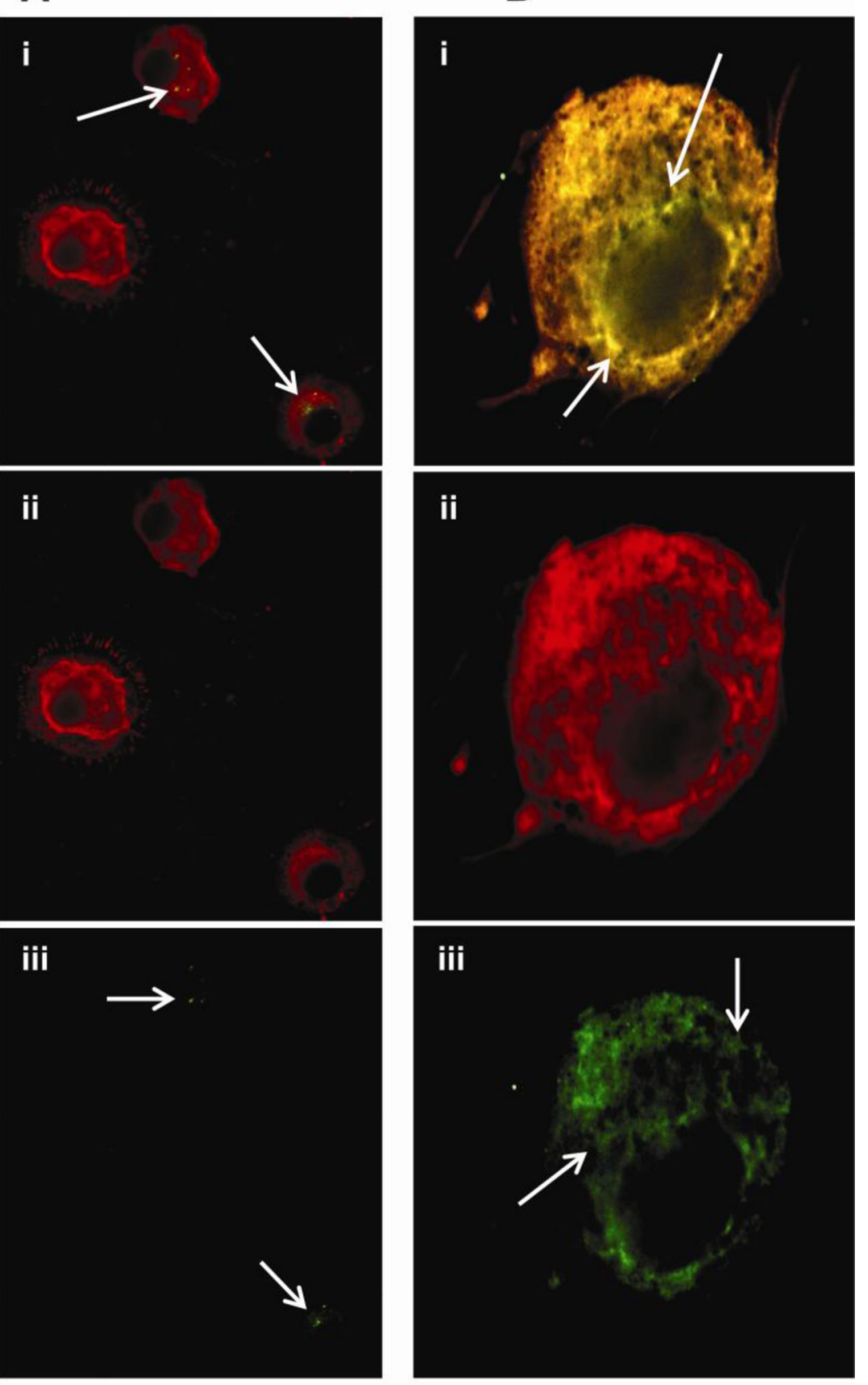

Figure 4.

Intracellular uptake of liposomes containing fasudil by (A) rat alveolar macrophages, and (B) rat pulmonary arterial smooth muscle cells. (i) overlay; (ii) TRITC channel; and (iii) FITC channel. 

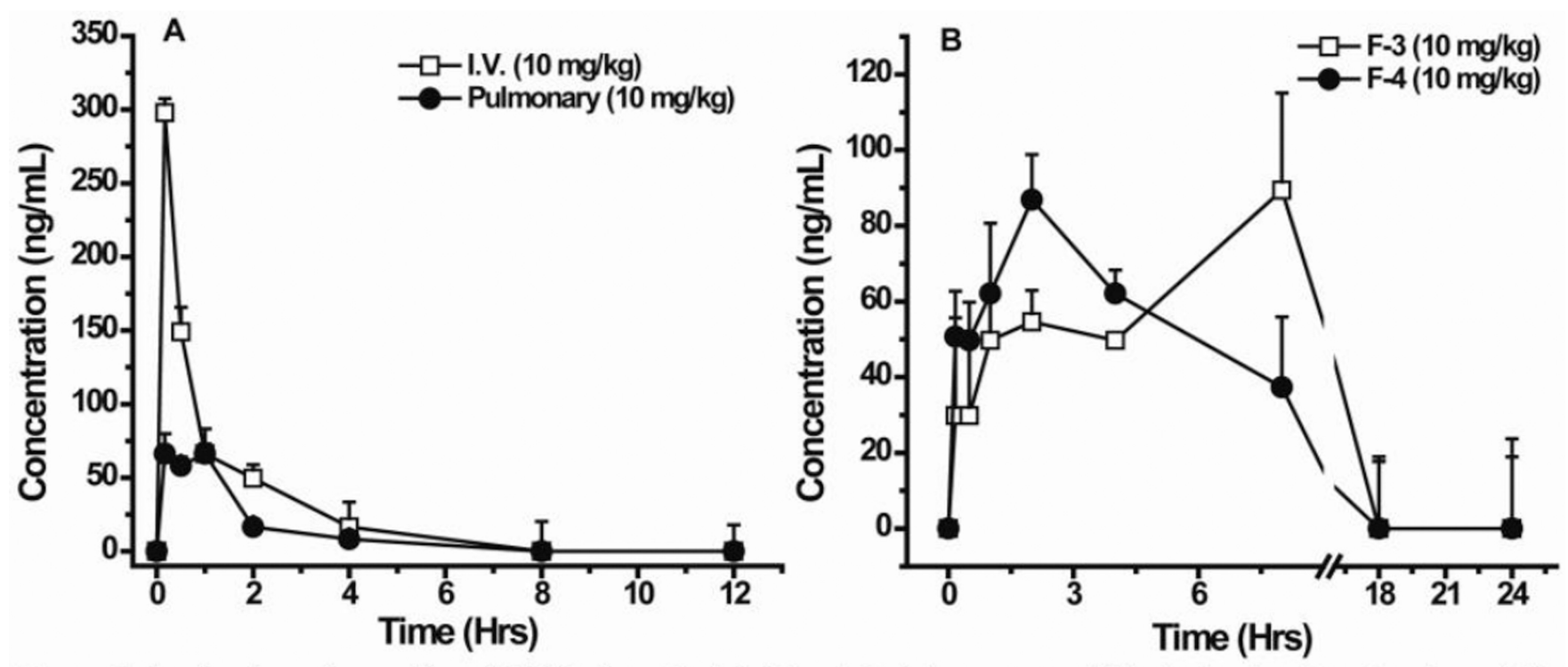

Figure 5.

In-vivo absorption profiles: (A) Plain fasudil administered via intravenous and intratracheal routes at a dose of $10 \mathrm{mg} / \mathrm{kg}$, and (B) Liposomal fasudil formulations administered intratracheally at a dose of $10 \mathrm{mg} / \mathrm{kg}$. Data represent mean \pm standard deviation $(\mathrm{n}=6-8)$. 

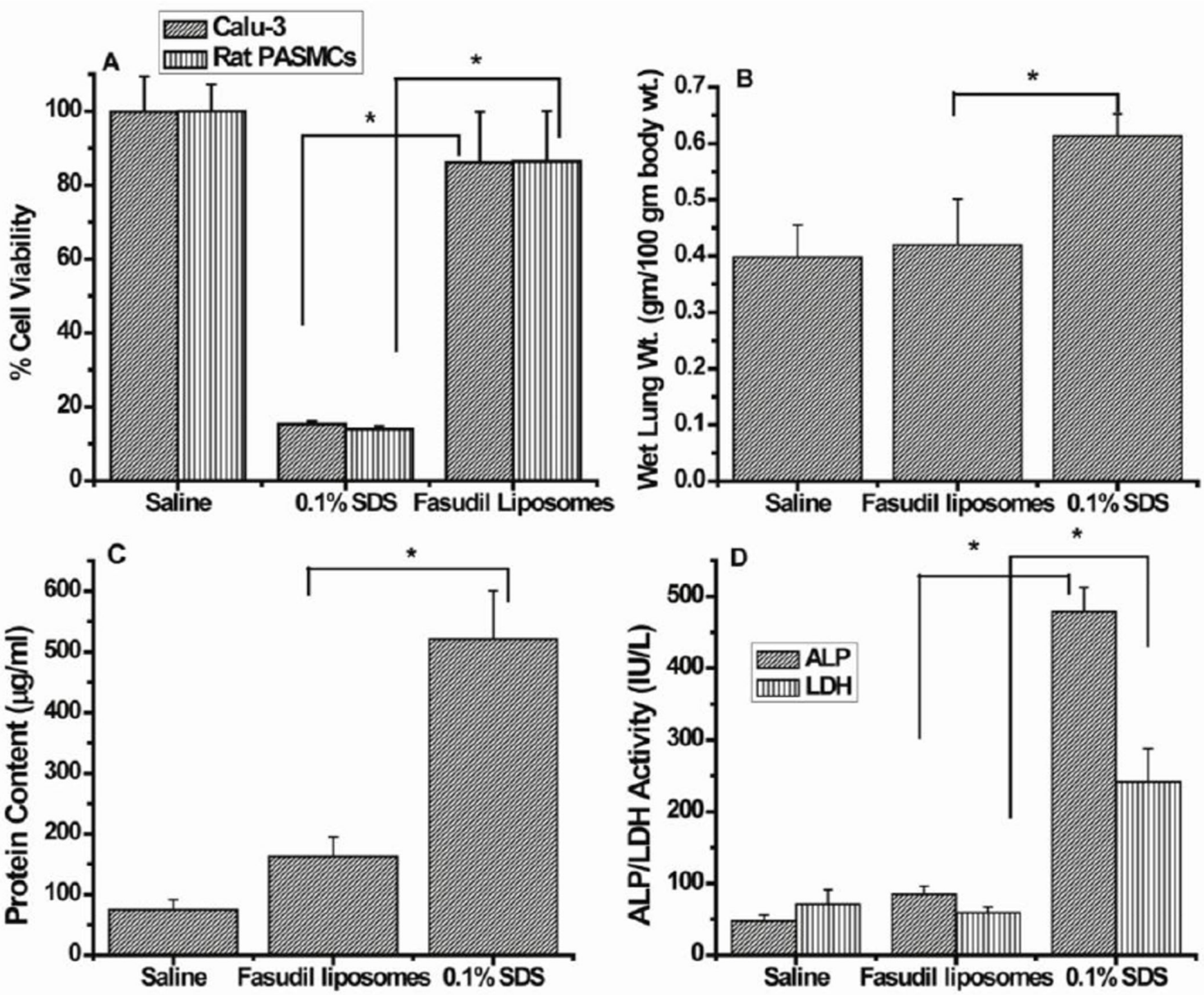

Figure 6.

Safety studies (A) cytotoxicity studies of fasudil liposomes $(100 \mu \mathrm{M})$ in human bronchial epithelial (Calu-3) and rat pulmonary arterial smooth muscle (PASM) cells for $24 \mathrm{hrs}$ ( $\mathrm{n}=$ 8). Effect of the formulation on the (B) wet lung weight, (C) total protein content, and (D) levels of injury markers in bronchioalveolar lavage (BAL) fluid. Data represent mean $\nabla$ s.d. $(\mathrm{n}=4)$, *means are significantly different $(\mathrm{p}<0.05), 0.1 \%$ SDS treatment is compared with fasudil liposomes. 

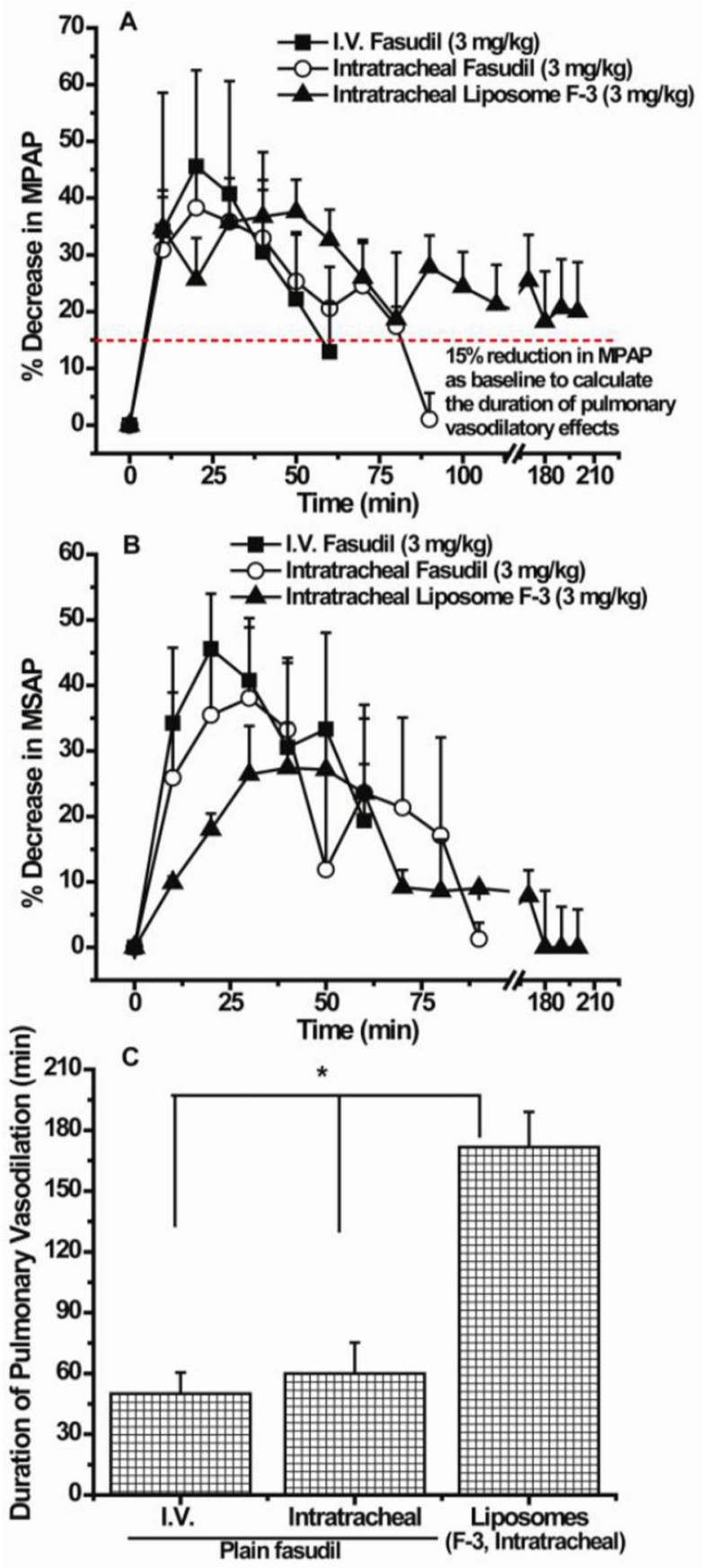

Figure 7.

Hemodynamic efficacy of the formulations in MCT induced PAH rats in reducing (A) mean pulmonary arterial pressure (MPAP) [horizontal dashed line (----) represents $15 \%$ reduction in MPAP] and (B) mean systemic arterial pressure upon administration of plain and liposomal fasudil at a single dose of $3 \mathrm{mg} / \mathrm{kg}$. (C) Duration of pulmonary vasodilatory effects calculated using 15\% reduction in MPAP as the baseline at the start and end points of MPAP recording. Data represent mean \pm standard deviation $(\mathrm{n}=4-6)$. *Means are significantly different from each other $(p<0.05)$. 


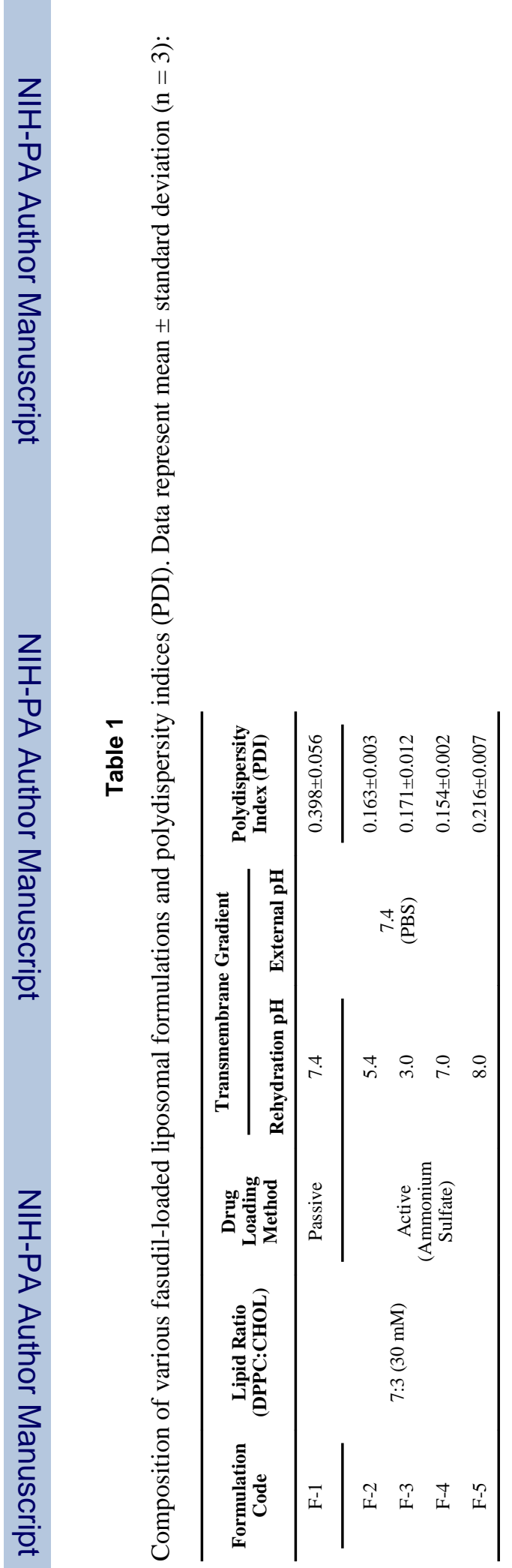


Table 2

Physicochemical characterization of fasudil loaded liposomes before and after nebulization with the microsprayer:

\begin{tabular}{ccccc}
\hline Nebulization & Size (nm) & PDI & $\begin{array}{c}\text { Zeta } \\
\text { Potential } \\
(\mathbf{m V})\end{array}$ & $\begin{array}{c}\text { Entrapment } \\
\text { Efficiency } \\
(\boldsymbol{\%})\end{array}$ \\
\hline No & $180.067 \pm 5.464$ & $0.92 \pm 0.004$ & $-17.3 \pm 0.476$ & $63.69 \pm 1.748$ \\
Yes & $180.567 \pm 1.95$ & $0.85 \pm 0.012$ & $-16.9 \pm 0.929$ & $63.18 \pm 0.891$ \\
\hline
\end{tabular}




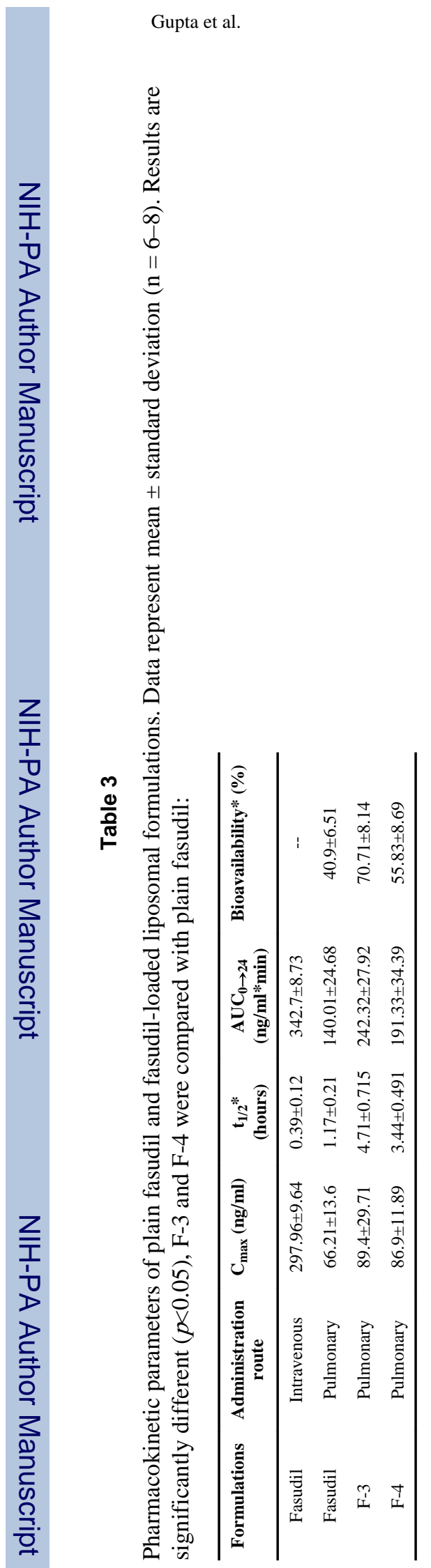

J Control Release. Author manuscript; available in PMC 2014 April 28. 